\title{
A Method to Construct Generalized Fibonacci Sequences
}

\author{
Adalberto García-Máynez ${ }^{1}$ and Adolfo Pimienta Acosta ${ }^{2}$ \\ ${ }^{1}$ Instituto de Matemáticas, Universidad Nacional Autónoma de México, Area de la Investigación Científica Circuito Exterior, \\ Ciudad Universitaria Coyoacán, 04510 México, DF, Mexico \\ ${ }^{2}$ Departamento de Matemáticas, Universidad Autónoma Metropolitana, Prolongación Canal de Miramontes No. 3855, \\ Colonia Ex-Hacienda San Juan de Dios, Delegación Tlalpan, 14387 México, DF, Mexico
}

Correspondence should be addressed to Adolfo Pimienta Acosta; pimienta331@hotmail.com

Received 17 September 2015; Accepted 6 January 2016

Academic Editor: Allan C. Peterson

Copyright (C) 2016 A. García-Máynez and A. P. Acosta. This is an open access article distributed under the Creative Commons Attribution License, which permits unrestricted use, distribution, and reproduction in any medium, provided the original work is properly cited.

\begin{abstract}
The main purpose of this paper is to study the convergence properties of Generalized Fibonacci Sequences and the series of partial sums associated with them. When the proper values of an $s \times s$ real matrix $A$ are real and different, we give a necessary and sufficient condition for the convergence of the matrix sequence $A, A^{2}, A^{3}, \ldots$ to a matrix $B$.
\end{abstract}

\section{Introduction}

The Fibonacci Sequence

$$
0,1,1,2,3,5,8,13,21, \ldots
$$

is an interesting numerical sequence that occurs quite frequently in many parts of nature. This sequence has a special feature; every element of this sequence, starting from the third, is the sum of its two predecessors and can be generated recursively by the formula

$$
x_{n+2}=x_{n}+x_{n+1} \text {. }
$$

It is clear that we need the first two terms $x_{0}=0, x_{1}=1$ and the recursive formula to define the sequence.

If we want to know the term $x_{k}$ without constructing the previous terms, we can use the unexplainable formula (see [1]):

$$
x_{k}=\frac{1}{\sqrt{5}}\left[\left(\frac{1+\sqrt{5}}{2}\right)^{k}-\left(\frac{1-\sqrt{5}}{2}\right)^{k}\right] \text {. }
$$

What do the irrational numbers $\sqrt{5}$ have to do with the original sequence?
The so-called Golden ratio $\lambda=(1+\sqrt{5}) / 2$ appears in nature very frequently. It is also considered the most esthetic ratio between the basis and height of a rectangle:

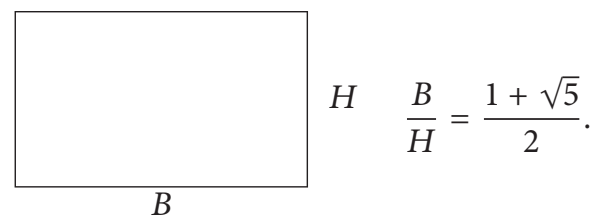

If we replace the recursive formula by

$$
2 x_{n+2}=x_{n}+x_{n+1} \text {, }
$$

we obtain a new sequence $0,1,1 / 2,3 / 4,5 / 8,11 / 16,21 / 32, \ldots$. and this sequence is no longer divergent; in fact, it converges to $2 / 3$.

To define a Generalized Fibonacci Sequence, we fix a natural number $s$ and two elements

$$
\begin{gathered}
\left(x_{0}, x_{1}, \ldots, x_{s-1}\right), \\
\left(a_{0}, a_{1}, \ldots, a_{s-1}\right)
\end{gathered}
$$

in the Euclidean space $\mathbb{R}^{s}$. The recursive formula is

$$
x_{k+s}=a_{0} x_{k}+a_{1} x_{k+1}+\cdots+a_{s-1} x_{k+s-1} .
$$


The main purpose of this paper is to study the convergence properties of Generalized Fibonacci Sequences and the series of partial sums associated with them. When the proper values of an $s \times s$ real matrix $A$ are real and different, we give a necessary and sufficient condition for the convergence of the matrix sequence $I, A, A^{2}, A^{3}, \ldots$ to a matrix $B$ : we say $A^{k} \rightarrow B$ if for every ordered pair $(i, j)$, where $i, j \in\{1,2, \ldots, s\}$, the sequence of the $(i, j)$-entries of $A^{k}$ converges to the $(i, j)$ entry of $B$. As a particular case, we study when do we have the convergence of the powers $\varphi, \varphi^{2}, \varphi^{3}, \ldots$ of a Moebius transformation to a constant function.

Then we would like to list the four published monographs about generalized Fibonacci sequences [1-4] and several more specialized articles [5-8].

\section{Main Results}

Consider a Generalized Fibonacci Sequence (GFS) with initial terms $x_{0}, x_{1}, \ldots, x_{s-1}$ and recursive formula (7). Define the matrices $s \times s$ :

$$
\begin{aligned}
C & =\left(\begin{array}{ccccc}
0 & 1 & 0 & \cdots & 0 \\
0 & 0 & 1 & \cdots & 0 \\
\vdots & \vdots & \vdots & \vdots & \vdots \\
0 & 0 & 0 & \cdots & 1 \\
a_{0} & a_{1} & a_{2} & \cdots & a_{s-1}
\end{array}\right), \\
D_{k} & =\left(\begin{array}{cccc}
x_{k} & x_{k+1} & \cdots & x_{k+s-1} \\
x_{k+1} & x_{k+2} & \cdots & x_{k+s} \\
\vdots & \vdots & \vdots & \vdots \\
x_{k+s-1} & x_{k+s} & \cdots & x_{k+2 s-2}
\end{array}\right), k \in \mathbb{N} \cup\{0\} .
\end{aligned}
$$

The characteristic polynomial of the matrix $C$ is

$$
\varphi(x)=x^{s}-a_{s-1} x^{s-1}-a_{s-2} x^{s-2}-\cdots-a_{0} .
$$

Suppose all the roots $\lambda_{1}, \lambda_{2}, \ldots, \lambda_{s}$ of $\varphi(x)$ are real and pairwise different; that is, $\lambda_{i} \neq \lambda_{j}$, for $i \neq j$. Consider Vandermonde's matrix:

$$
V=\left(\begin{array}{cccc}
1 & 1 & \cdots & 1 \\
\lambda_{1} & \lambda_{2} & \cdots & \lambda_{s} \\
\lambda_{1}^{2} & \lambda_{2}^{2} & \cdots & \lambda_{s}^{2} \\
\vdots & \vdots & \vdots & \vdots \\
\lambda_{1}^{s-1} & \lambda_{2}^{s-1} & \cdots & \lambda_{s}^{s-1}
\end{array}\right) .
$$

Since $|V|= \pm \prod_{1 \leq i<j \leq s}\left(\lambda_{i}-\lambda_{j}\right)$, we deduce that $|V| \neq 0$ and, hence, $V$ is invertible. We need the following matrix relation.

Theorem 1. $V$ and $C$ are related by the following formula:

$$
V E V^{-1}=C
$$

where $E$ is the diagonal matrix:

$$
E=\left(\begin{array}{cccc}
\lambda_{1} & 0 & \cdots & 0 \\
0 & \lambda_{2} & \cdots & 0 \\
\vdots & \vdots & \ddots & \vdots \\
0 & 0 & \cdots & \lambda_{s}
\end{array}\right)
$$

Proof. Let $v_{1}, v_{2}, \ldots, v_{s} \in \mathbb{R}^{s}$ be the proper vectors of the matrix $C$. We have then $v_{i} C=\lambda_{i} v_{i}$ for each $i=1,2, \ldots, s$. If $w=v_{1}+v_{2}+\cdots+v_{s}$ and $k \in \mathbb{N}$, we have $w C^{k}=$ $\lambda_{1}^{k} v_{1}+\lambda_{2}^{k} v_{2}+\cdots+\lambda_{s}^{k} v_{s}, k=0,1, \ldots, s-1$. Since $|V| \neq 0$, we deduce that the vectors $w, w C, w C^{2}, \ldots, w C^{s-1}$ are linearly independent and hence they constitute a basis for $\mathbb{R}^{s}$. Calling $\mathscr{B}=\left\{v_{1}, v_{2}, \ldots v_{s}\right\}, \mathscr{B}^{\prime}=\left\{w, w C, \ldots, w C^{s-1}\right\}$, we have $V=m\left(I d, \mathscr{B}^{\prime}, \mathscr{B}\right)$. On the other hand, consider the linear transformation $T: \mathbb{R}^{s} \rightarrow \mathbb{R}^{s}$ defined by the formula $T(v)=$ $v C$. Clearly,

$$
C=m\left(T, \mathscr{B}^{\prime}, \mathscr{B}^{\prime}\right)
$$

Therefore,

$$
\begin{aligned}
C & =m\left(T, \mathscr{B}^{\prime}, \mathscr{B}^{\prime}\right) \\
& =m\left(I d, \mathscr{B}^{\prime}, \mathscr{B}\right) m(T, \mathscr{B}, \mathscr{B}) m\left(I d, \mathscr{B}, \mathscr{B}^{\prime}\right) \\
& =V E V^{-1} .
\end{aligned}
$$

In the next theorem, we relate $V$ and $C$ with $D_{k}$.

Theorem 2. One has the following formulas:
(a) $C D_{k}=D_{k+1}$ and $C^{k} D_{0}=D_{k}$ for every $k \in \mathbb{N}$.
(b) $D_{k}=V E^{k} V^{-1} D_{0}$ for every $k \in \mathbb{N}$.

Proof. (a) It is straightforward.

(b) Using (36), we have

$$
D_{k}=C^{k} D_{0}=\left(V E V^{-1}\right)^{k} D_{0}=V E^{k} V^{-1} D_{0} .
$$

Using the formula $D_{k}=V E^{k} V^{-1} D_{0}$, we obtain any member of the corresponding Generalized Fibonacci Sequence.

Theorem 3. Consider

$$
x_{k}=\frac{(-1)^{s-1}}{|V|}\left|\begin{array}{ccccc}
\lambda_{1}^{k} & \lambda_{2}^{k} & \cdots & \lambda_{s}^{k} & 0 \\
1 & 1 & \cdots & 1 & x_{0} \\
\lambda_{1} & \lambda_{2} & \cdots & \lambda_{s} & x_{1} \\
\vdots & \vdots & \ddots & \vdots & \vdots \\
\lambda_{1}^{s-1} & \lambda_{2}^{s-1} & \cdots & \lambda_{s}^{s-1} & x_{s-1}
\end{array}\right| .
$$


Proof. The first row of the matrix $V E^{k}$ is the following: $\left(\lambda_{1}^{k}, \lambda_{2}^{k}, \ldots, \lambda_{s}^{k}\right)$. The first column of the matrix $V^{-1} D_{0}$ is

$$
\frac{1}{|V|}\left(\begin{array}{c}
\sum_{j=1}^{s} V_{j 1} x_{j-1} \\
\sum_{j=1}^{s} V_{j 2} x_{j-1} \\
\vdots \\
\sum_{j=1}^{s} V_{j s} x_{j-1}
\end{array}\right),
$$

where $V_{i j}$ is the cofactor of the entry of $V$ in the $(i, j)$ position. $x_{k}$ is the entry in the $(1,1)$ position of the matrix $D_{k}$. Therefore,

$$
\begin{aligned}
x_{k} & =\frac{1}{|V|}\left[\lambda_{1}^{k} \sum_{j=1}^{s} V_{j 1} x_{j-1}+\lambda_{2}^{k} \sum_{j=1}^{s} V_{j 2} x_{j-1}+\cdots\right. \\
& \left.+\lambda_{s}^{k} \sum_{j=1}^{s} V_{j s} x_{j-1}\right] .
\end{aligned}
$$

The expression inside the square brackets coincides with

$$
(-1)^{s-1}\left|\begin{array}{ccccc}
\lambda_{1}^{k} & \lambda_{2}^{k} & \cdots & \lambda_{s}^{k} & 0 \\
1 & 1 & \cdots & 1 & x_{0} \\
\lambda_{1} & \lambda_{2} & \cdots & \lambda_{s} & x_{1} \\
\vdots & \vdots & \vdots & \vdots & \vdots \\
\lambda_{1}^{s-1} & \lambda_{2}^{s-1} & \cdots & \lambda_{s}^{s-1} & x_{s-1}
\end{array}\right| .
$$

To see this, develop this determinant by the first row and the last column. The coefficient of $\lambda_{i}^{k} x_{j-1}$ is then

$$
\begin{gathered}
(-1)^{i-1}(-1)^{s+j}\left|\begin{array}{ccccc}
1 & 1 & \cdots & 1 & 1 \\
\lambda_{1} & \cdots & \lambda_{i-1} \lambda_{i+1} & \cdots & \lambda_{s} \\
\vdots & \vdots & \vdots & \vdots & \vdots \\
\lambda_{1}^{j-1} & \cdots & \lambda_{i-1}^{j-1} \lambda_{i+1}^{j-1} & \cdots & \lambda_{s}^{j-1} \\
\lambda_{1}^{j+1} & \cdots & \lambda_{i-1}^{j+1} \lambda_{i+1}^{j+1} & \cdots & \lambda_{s}^{j+1} \\
\vdots & \vdots & \ddots & \vdots & \vdots \\
\lambda_{1}^{s-1} & \cdots & \lambda_{i-1}^{s-1} \lambda_{i+1}^{s-1} & \cdots & \lambda_{s}^{s-1}
\end{array}\right| \\
=(-1)^{s-1} V_{j i} .
\end{gathered}
$$

This completes the proof.

In the particular case $k=2$, we obtain

$$
x_{k}=-\frac{1}{\lambda_{2}-\lambda_{1}}\left|\begin{array}{ccc}
\lambda_{1}^{k} & \lambda_{2}^{k} & 0 \\
1 & 1 & x_{0} \\
\lambda_{1} & \lambda_{2} & x_{1}
\end{array}\right| \text {. }
$$

If we further assume that $x_{0}=0$ and $x_{1}=1$, we obtain

$$
x_{k}=\frac{1}{\lambda_{1}-\lambda_{2}}\left(\lambda_{1}^{k}-\lambda_{2}^{k}\right) \text {. }
$$

In the original Fibonacci sequence, we have

$$
C=\left(\begin{array}{ll}
0 & 1 \\
1 & 1
\end{array}\right)
$$

and hence $\varphi(x)=x^{2}-x-1$. The roots of this polynomial are $\lambda_{1}=(1+\sqrt{5}) / 2$ and $\lambda_{2}=(1-\sqrt{5}) / 2$. We justify then the mythical formula $x_{k}=(1 / \sqrt{5})\left[((1+\sqrt{5}) / 2)^{k}-((1-\sqrt{5}) / 2)^{k}\right]$.

In the case $a_{0}=a_{1}=1 / 2$, we obtain

$$
\begin{aligned}
C & =\left(\begin{array}{cc}
0 & 1 \\
\frac{1}{2} & \frac{1}{2}
\end{array}\right), \\
\varphi(x) & =\left|\begin{array}{cc}
x & -1 \\
-\frac{1}{2} & x-\frac{1}{2}
\end{array}\right|=x^{2}-\frac{1}{2} x-\frac{1}{2} .
\end{aligned}
$$

The roots of $\varphi(x)$ are $\lambda_{1}=1$ and $\lambda_{2}=-1 / 2$. Hence, $x_{k}=$ $(2 / 3)\left[1-(-1 / 2)^{k}\right]$. It is now clear that this last GFS converges to $2 / 3$.

We give next a sufficient condition for the convergence of the series of a GFS.

Theorem 4 (main theorem). Suppose the roots of the characteristic polynomial $\varphi(x)$ of a GFS $\left\{x_{k}\right\}$ are pairwise different and all of them lie in the open interval $(-1,1)$. Then the series of $\left\{x_{k}\right\}$ converges to

$$
\frac{(-1)^{s-1}}{|V|}\left|\begin{array}{ccccc}
\frac{1}{1-\lambda_{1}} & \frac{1}{1-\lambda_{2}} & \cdots & \frac{1}{1-\lambda_{s}} & 0 \\
1 & 1 & \cdots & 1 & x_{0} \\
\lambda_{1} & \lambda_{2} & \cdots & \lambda_{s} & x_{1} \\
\vdots & \vdots & \vdots & \vdots & \vdots \\
\lambda_{1}^{s-1} & \lambda_{2}^{s-1} & \cdots & \lambda_{s}^{s-1} & x_{s-1}
\end{array}\right| .
$$

Proof. This is a consequence of the identity

$$
\begin{aligned}
& x_{0}+x_{1}+\cdots+x_{k}=\frac{(-1)^{s-1}}{|V|} \\
& \qquad \begin{array}{cccc}
1+\lambda_{1}+\lambda_{1}^{2}+\cdots+\lambda_{1}^{k-1} & \cdots & 1+\lambda_{s}+\lambda_{s}^{2}+\cdots+\lambda_{s}^{k-1} & 0 \\
1 & \cdots & 1 & x_{0} \\
\lambda_{1} & \cdots & \lambda_{s} & x_{1} \\
\vdots & \vdots & \vdots & \vdots \\
\lambda_{1}^{s-1} & \cdots & \lambda_{s}^{s-1} & x_{s-1}
\end{array} \mid
\end{aligned}
$$

and the convergence $\sum_{j=0}^{\infty} \lambda_{i}^{j}$ to $1 /\left(1-\lambda_{i}\right)$.

We give now two examples. 
Example 5. One has $s=3, x_{0}=x_{1}=0, x_{2}=1, a_{0}=1 / 6$, $a_{1}=-1$, and $a_{2}=11 / 6$.

The characteristic polynomial $\varphi(x)$ of the corresponding GFS is

$$
\begin{aligned}
\varphi(x) & =\left|\begin{array}{ccc}
x & -1 & 0 \\
0 & x & -1 \\
-\frac{1}{6} & 1 & x-\frac{11}{6}
\end{array}\right|=x^{3}-\frac{11}{6} x^{2}+x-\frac{1}{6} \\
& =(x-1)\left(x-\frac{1}{2}\right)\left(x-\frac{1}{3}\right) .
\end{aligned}
$$

Therefore, $\lambda_{1}=1, \lambda_{2}=1 / 2, \lambda_{3}=1 / 3$, and

$$
|V|=\left|\begin{array}{lll}
1 & 1 & 1 \\
1 & \frac{1}{2} & \frac{1}{3} \\
1 & \frac{1}{4} & \frac{1}{9}
\end{array}\right|=-\frac{1}{18} .
$$

Hence

$$
\begin{aligned}
x_{k} & =(-1)^{3-1}(-18)\left|\begin{array}{cccc}
1 & 2^{-k} & 3^{-k} & 0 \\
1 & 1 & 1 & 0 \\
1 & \frac{1}{2} & \frac{1}{3} & 0 \\
1 & \frac{1}{4} & \frac{1}{9} & 1
\end{array}\right| \\
& =-18\left|\begin{array}{ccc}
1 & 2^{-k} & 3^{-k} \\
1 & 1 & 1 \\
1 & \frac{1}{2} & \frac{1}{3}
\end{array}\right| .
\end{aligned}
$$

Clearly

$$
x_{k} \longrightarrow-18\left|\begin{array}{lll}
1 & 0 & 0 \\
1 & 1 & 1 \\
1 & \frac{1}{2} & \frac{1}{3}
\end{array}\right|=3
$$

Example 6. One has $s=3, x_{0}=x_{1}=0, x_{2}=1, a_{0}=$ $1 / 18, a_{1}=-11 / 8$, and $a_{2}=3 / 2$. In this case, $\varphi(x)=x^{3}-$ $(3 / 2) x^{2}+(11 / 18) x-1 / 18=(x-1 / 2)(x-1 / 3)(x-2 / 3)$. Therefore, $\lambda_{1}=1 / 2, \lambda_{2}=1 / 3, \lambda_{3}=2 / 3$, and $|V|=-1 / 108$. Hence,

$$
x_{k}=-108\left|\begin{array}{cccc}
\left(\frac{1}{2}\right)^{k} & \left(\frac{1}{3}\right)^{k} & \left(\frac{2}{3}\right)^{k} & 0 \\
1 & 1 & 1 & 0 \\
\frac{1}{2} & \frac{1}{3} & \frac{2}{3} & 0 \\
\frac{1}{4} & \frac{1}{9} & \frac{4}{9} & 1
\end{array}\right| \text {. }
$$

In this case, $x_{k} \rightarrow 0$, but

$$
\sum_{k=1}^{\infty} x_{k}=-108\left|\begin{array}{cccc}
\frac{1}{1-1 / 2} & \frac{1}{1-1 / 3} & \frac{1}{1-2 / 3} & 0 \\
1 & 1 & 1 & 0 \\
\frac{1}{2} & \frac{1}{3} & \frac{2}{3} & 0 \\
\frac{1}{4} & \frac{1}{9} & \frac{4}{9} & 1
\end{array}\right|=9 .
$$

Given $s$ different real numbers $\lambda_{1}, \lambda_{2}, \lambda_{2}, \ldots, \lambda_{s}$, we may construct, for every $\left(x_{0}, x_{1}, \ldots, x_{s-1}\right) \in \mathbb{R}^{s}$, a GFS; namely,

$$
x_{k}=\frac{(-1)^{s-1}}{\prod_{1 \leq j<i \leq s}\left(\lambda_{i}-\lambda_{j}\right)}\left|\begin{array}{ccccc}
\lambda_{1}^{k} & \lambda_{2}^{k} & \cdots & \lambda_{s}^{k} & 0 \\
1 & 1 & \cdots & 1 & x_{0} \\
\lambda_{1} & \lambda_{2} & \cdots & \lambda_{s} & x_{1} \\
\vdots & \vdots & \ddots & \vdots & \vdots \\
\lambda_{1}^{s-1} & \lambda_{2}^{s-1} & \cdots & \lambda_{s}^{s-1} & x_{s-1}
\end{array}\right| .
$$

The missing sequence $\left(a_{0}, a_{1}, \ldots, a_{s-1}\right)$ may be obtained from the coefficients of the polynomial:

$$
\varphi(x)=\left(x-\lambda_{1}\right)\left(x-\lambda_{2}\right) \cdots\left(x-\lambda_{s}\right) \text {. }
$$

For instance, $a_{s-1}=\lambda_{1}+\lambda_{2}+\cdots+\lambda_{s}$ and $a_{s-2}=$ $-\sum_{1 \leq j<i \leq s} \lambda_{i} \lambda_{j}$

With the help of this remark, we prove the following.

Theorem 7. Let $\left(a_{i j}\right)$ be an invertible $s \times s$ matrix. Suppose the characteristic polynomial $\varphi(x)$ of A factors into the form:

$$
\varphi(x)=\left(x-\lambda_{1}\right)\left(x-\lambda_{2}\right) \cdots\left(x-\lambda_{s}\right),
$$

where $\lambda_{1}, \lambda_{2}, \ldots, \lambda_{s}$ are pairwise different real numbers. Let $a_{i j}^{(k)}$ be the entry in the $(i, j)$ position of the matrix $A^{k}$. Then

$$
a_{i j}^{(k)}=\frac{(-1)^{s-1}}{|V|}\left|\begin{array}{ccccc}
\lambda_{1}^{k} & \lambda_{2}^{k} & \cdots & \lambda_{s}^{k} & 0 \\
1 & 1 & \cdots & 1 & a_{i j}^{(0)} \\
\lambda_{1} & \lambda_{2} & \cdots & \lambda_{s} & a_{i j}^{(1)} \\
\lambda_{1}^{2} & \lambda_{2}^{2} & \cdots & \lambda_{s}^{2} & a_{i j}^{(2)} \\
\vdots & \vdots & \vdots & \vdots & \vdots \\
\lambda_{1}^{s-1} & \lambda_{2}^{s-1} & \cdots & \lambda_{s}^{(s-1)} & a_{i j}^{(s-1)}
\end{array}\right|
$$

Proof. Let $\left(x_{k}\right)$ be the GFS determined by (36). It is clear that $x_{k}=a_{i j}^{(k)}$ for $k \leq s-1$. Proceeding by induction, suppose $x_{t}=a_{i j}^{(t)}$ for every $t \leq k$, where $k \geq s-1$. We clearly have

$$
\begin{aligned}
a_{i j}^{(k+1)} & =\left(a_{i 1}^{(k)}, a_{i 2}^{(k)}, \ldots, a_{i s}^{(k)}\right) \cdot\left(a_{1 j}^{(1)}, a_{2 j}^{(1)}, \ldots, a_{s j}^{(1)}\right) \\
& =a_{i 1}^{(k)} a_{1 j}+a_{i 2}^{(k)} a_{2 j}+\cdots+a_{i s}^{(k)} a_{s j} .
\end{aligned}
$$


Be induction, the terms $a_{i 1}^{(k)}, \ldots, a_{i s}^{(k)}$ may be obtained using determinants of type (36). We consider the last columns of determinants (36) and we obtain

$$
\begin{aligned}
& a_{1 j}\left(\begin{array}{c}
0 \\
a_{i 1}^{(0)} \\
a_{i 1}^{(1)} \\
\vdots \\
a_{i 1}^{(s-1)}
\end{array}\right)+a_{2 j}\left(\begin{array}{c}
0 \\
a_{i 2}^{(0)} \\
a_{i 2}^{(1)} \\
\vdots \\
a_{i 2}^{(s-1)}
\end{array}\right)+\cdots \\
& =\left(\begin{array}{c}
0 \\
a_{i j}^{(1)} \\
a_{i j}^{(2)} \\
\vdots \\
a_{i j}^{(s)}
\end{array}\right) .
\end{aligned}
$$

Therefore

$$
a_{i j}^{(k+1)}=\frac{(-1)^{s-1}}{|\Delta|}\left|\begin{array}{ccccc}
\lambda_{1}^{k} & \lambda_{2}^{k} & \cdots & \lambda_{s}^{k} & 0 \\
1 & 1 & \cdots & 1 & a_{i j}^{(1)} \\
\lambda_{1} & \lambda_{2} & \cdots & \lambda_{s} & a_{i j}^{(2)} \\
\lambda_{1}^{2} & \lambda_{2}^{2} & \cdots & \lambda_{s}^{2} & a_{i j}^{(3)} \\
\vdots & \vdots & \vdots & \vdots & \vdots \\
\lambda_{1}^{s-1} & \lambda_{2}^{s-1} & \cdots & \lambda_{s}^{(s-1)} & a_{i j}^{(s)}
\end{array}\right| .
$$

The GFS on the right side of this equation starts with $y_{0}=$ $a_{i j}^{(1)}, y_{1}=a_{i j}^{(2)}, \ldots, y_{s-1}=a_{i j}^{(s)}$ and has the same proper values of the GFS $\left(x_{k}\right)$. Hence $a_{i j}^{(k+1)}=y_{k}=x_{k+1}$ and the proof is complete.

As an exercise, we calculate the powers of a $2 \times 2$ matrix:

$$
A=\left(\begin{array}{ll}
a & b \\
h & k
\end{array}\right), \quad \text { where } \Delta=a k-h b \neq 0 .
$$

In this case we have $a_{11}^{(0)}=1, a_{12}^{(0)}=0, a_{21}^{(0)}=0, a_{22}^{(0)}=1$, $a_{11}^{(1)}=a, a_{12}^{(1)}=b, a_{21}^{(1)}=h, a_{22}^{(1)}=k$. Hence

$$
a_{i j}^{(t)}=-\frac{1}{\left|\begin{array}{cc}
1 & 1 \\
\lambda_{1} & \lambda_{2}
\end{array}\right|}\left|\begin{array}{ccc}
\lambda_{1}^{t} & \lambda_{2}^{t} & 0 \\
1 & 1 & a_{i j}^{(0)} \\
\lambda_{1} & \lambda_{2} & a_{i j}^{(1)}
\end{array}\right| .
$$

Therefore

$$
\begin{aligned}
& a_{11}^{(t)}=\frac{1}{\lambda_{1}-\lambda_{2}}\left|\begin{array}{ccc}
\lambda_{1}^{t} & \lambda_{2}^{t} & 0 \\
1 & 1 & 1 \\
\lambda_{1} & \lambda_{2} & a
\end{array}\right| ; \\
& a_{12}^{(t)}=\frac{1}{\lambda_{1}-\lambda_{2}}\left|\begin{array}{ccc}
\lambda_{1}^{t} & \lambda_{2}^{t} & 0 \\
1 & 1 & 0 \\
\lambda_{1} & \lambda_{2} & b
\end{array}\right| ; \\
& a_{21}^{(t)}=\frac{1}{\lambda_{1}-\lambda_{2}}\left|\begin{array}{ccc}
\lambda_{1}^{t} & \lambda_{2}^{t} & 0 \\
1 & 1 & 0 \\
\lambda_{1} & \lambda_{2} & h
\end{array}\right| ; \\
& a_{22}^{(t)}=\frac{1}{\lambda_{1}-\lambda_{2}}\left|\begin{array}{ccc}
\lambda_{1}^{t} & \lambda_{2}^{t} & 0 \\
1 & 1 & 1 \\
\lambda_{1} & \lambda_{2} & k
\end{array}\right|,
\end{aligned}
$$

where $\lambda_{1}=(1 / 2)\left[(a+k)-\sqrt{(a-k)^{2}+4 h b}\right]$ and $\lambda_{2}=$ $(1 / 2)\left[(a+k)+\sqrt{(a-k)^{2}+4 h b}\right]$. If $\lambda_{1}=1$ and $\left|\lambda_{2}\right|<1$, we have

$$
A^{t} \longrightarrow \frac{1}{1-\lambda_{2}}\left(\begin{array}{cc}
a-\lambda_{2} & b \\
h & k-\lambda_{2}
\end{array}\right) \text {. }
$$

This limit matrix has determinant $=0$. So in the case of a Moebius transformation

$$
\varphi(z)=\frac{a+b z}{h+k z}, \quad a k \neq h b
$$

we have the following corollary.

Corollary 8. If 1 is a proper value of the matrix $A=\left(\begin{array}{ll}a & b \\ h & k\end{array}\right)$ and if $|a k-h b|<1$, then the powers $\varphi^{t}$ converge to the constant $\operatorname{map} \phi(z)=\left(a-\lambda_{2}\right) / h\left(1-\lambda_{2}\right)=b /\left(1-\lambda_{2}\right)\left(k-\lambda_{2}\right)$.

\section{Conflict of Interests}

The authors declare that there is no conflict of interests regarding the publication of this paper.

\section{References}

[1] A. F. Horadam, "A generalized Fibonacci sequence," The American Mathematical Monthly, vol. 68, no. 5, pp. 455-459, 1961.

[2] D. V. Jaiswal, "On a generalized Fibonacci sequence," Labdev: Journal of Science and Technology. Part A, vol. 7, pp. 67-71, 1969.

[3] S. P. Pethe and C. N. Phadte, "A generalized Fibonacci sequence," Applications of Fibonacci Numbers, vol. 5, pp. 465-472, 1992.

[4] D. A. Wolfram, "Solving generalized Fibonacci recurrences," The Fibonacci Quarterly, vol. 36, no. 2, pp. 129-145, 1998.

[5] G. Sburlati, "Generalized Fibonacci sequences and linear congruences," The Fibonacci Quarterly, vol. 40, no. 5, pp. 446-452, 2002. 
[6] G.-Y. Lee, S.-G. Lee, J.-S. Kim, and H.-K. Shin, “The Binet formula and representations of k-generalized Fibonacci numbers," The Fibonacci Quarterly, vol. 39, no. 2, pp. 158-164, 2001.

[7] G. Y. Lee, S. G. Lee, and H. G. Shin, "On the k-generalized Fibonacci matrix $Q_{k}$," Linear Algebra and Its Applications, vol. 251, pp. 73-88, 1997.

[8] S. T. Klein, "Combinatorial representation of generalized Fibonacci numbers," The Fibonacci Quarterly, vol. 29, no. 2, pp. 124-131, 1991. 


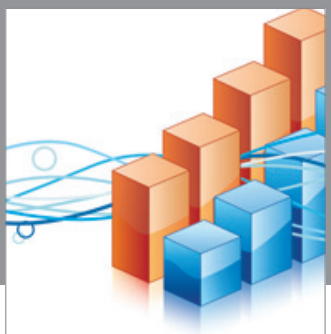

Advances in

Operations Research

vatem alat4

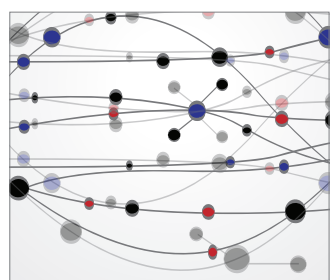

\section{The Scientific} World Journal
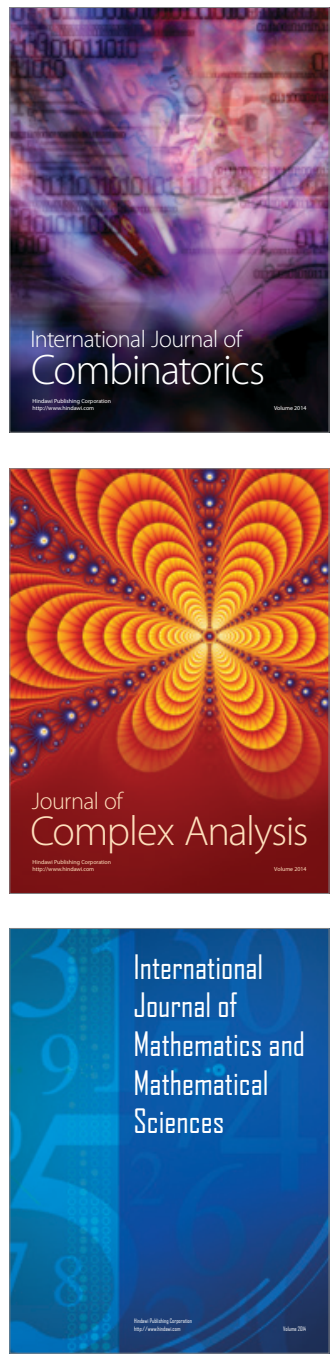
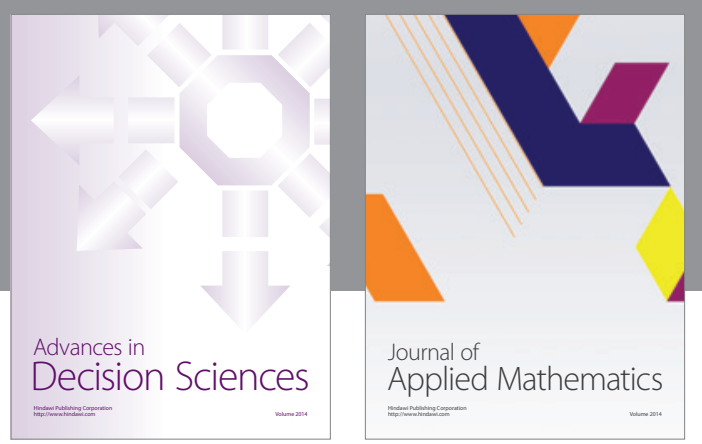

Algebra

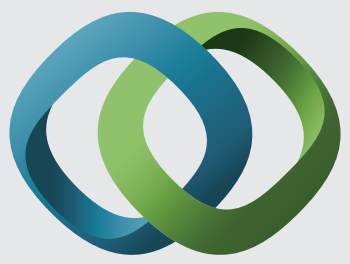

\section{Hindawi}

Submit your manuscripts at

http://www.hindawi.com
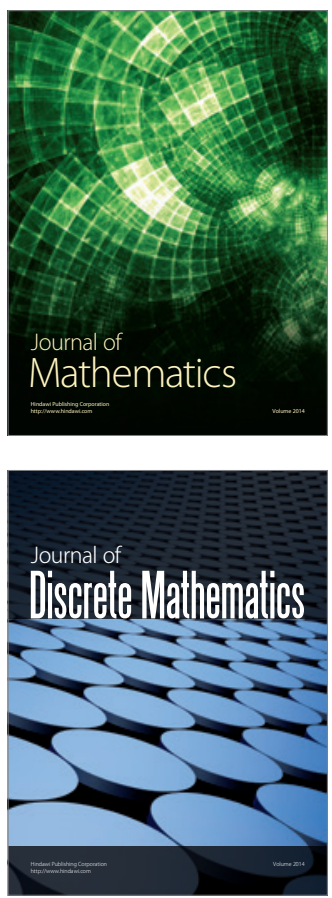

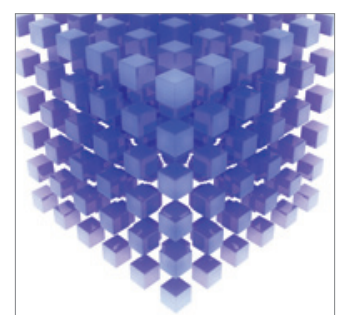

Mathematical Problems in Engineering
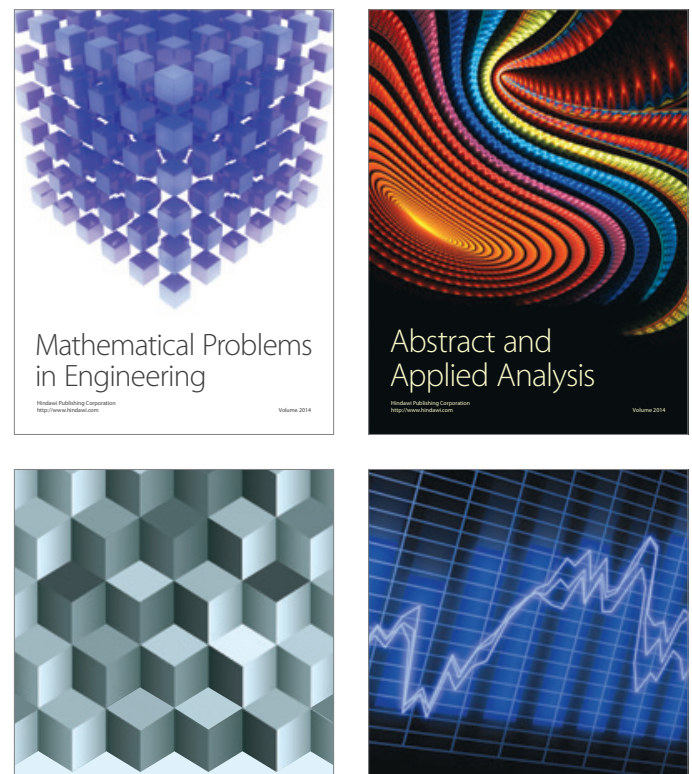

Journal of

Function Spaces

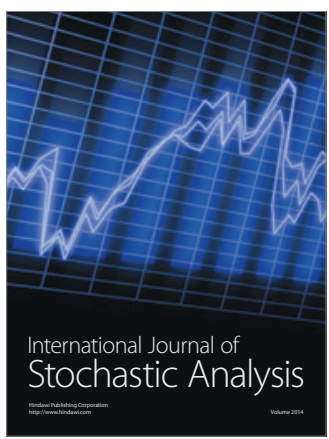

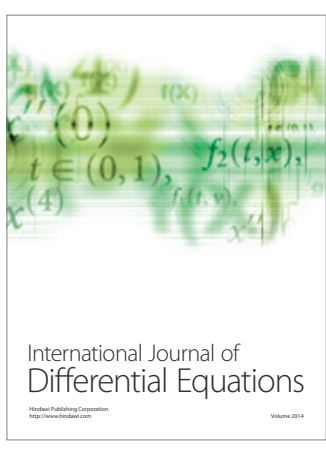
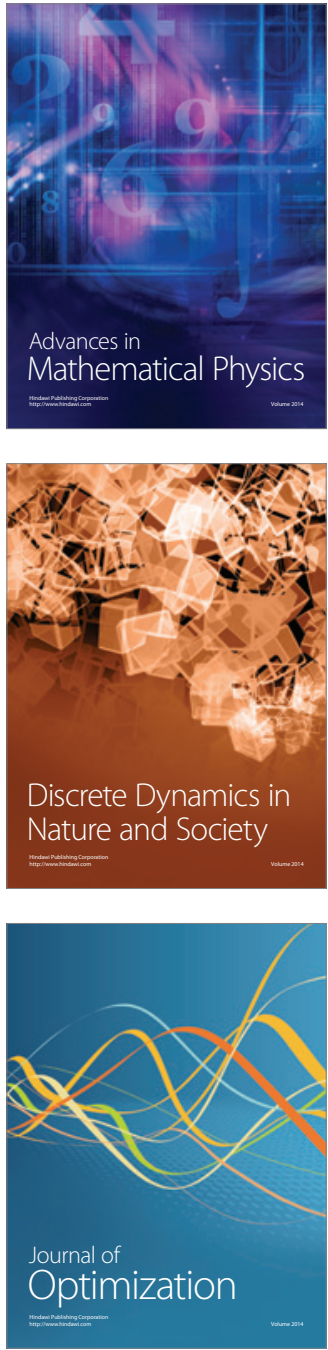